\title{
Novel adsorbent from sago-grafted silica for removal of methylene blue
}

\author{
International Journal of Environmental Science and Technology \\ pp 1-12 | Cite as \\ - Y. Rajan (1) \\ - Z. Ngaini (1) Email author (nzainab@unimas.my)View author's OrcID profile \\ (View OrcID profile) \\ - R. Wahi (1)
}

1. Faculty of Resource Science and Technology, Universiti Malaysia Sarawak, , Kota Samarahan, Sarawak, Malaysia

Original Paper

First Online: 09 October 2018

- 10 Downloads

\begin{abstract}
Eco-friendly and low-cost adsorbent prepared from sago waste was investigated for the removal of methylene blue. Sago "hampas," an abundant waste from sago industries, was transformed into activated carbon followed by chemical grafting with silica from activated rice husk in the presence of 3-(triethoxysilyl)-propylamine to afford sago-grafted silica 80:20 (wt/wt\%) and 50:50 (wt/wt\%). The physicochemical properties of the adsorbents were characterized, and their effectiveness in removing methylene blue was studied based on initial concentration (2-10 $\mathrm{mg} / \mathrm{L}$ ), adsorbent dosage (0.02-0.1 g and 0.1-0.5 g), and temperature $\left(30^{\circ} \mathrm{C}, 40^{\circ} \mathrm{C}, 50^{\circ} \mathrm{C}\right)$. Sagografted silica 80:20 showed excellent mesoporous properties and better adsorption capacity (86.43\%) compared to sago-activated carbon (74.78\%) and sago-grafted silica 50:50 (39.56\%). The adsorption of methylene blue employing both sago-activated carbon and sago-grafted silica 80:20 was following pseudo-second-order kinetics model with Langmuir isotherm regression coefficient $>0.9$, which indicate a monolayer adsorption. The maximum adsorption capacity of sago-activated carbon and sago-grafted silica 80:20 were $7.69 \mathrm{mg} / \mathrm{g}$ and $10.31 \mathrm{mg} / \mathrm{g}$, respectively. Sagografted silica 80:20 is a potential low-cost natural sorbent which works best in the removal of methylene blue from environment.
\end{abstract}

\section{Keywords}

Sago hampas Rice husk activated Langmuir isotherm Mesoporous Adsorption

Editorial responsibility: $\mathrm{J}$ Aravind. 


\section{Electronic supplementary material}

The online version of this article ( https://doi.org/10.1007/s13762-018-2043-x (https://doi.org/10.1007/s13762-018-2043-x)) contains supplementary material, which is available to authorized users.

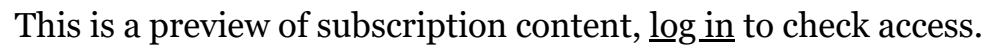

\section{Notes}

\section{Acknowledgements}

The authors would like to thank University Malaysia Sarawak and Ministry of Education for research fund, Openg Sago Research Chair

(Fo7(ORC16)/1222/2015(O3)).

\section{Supplementary material}

$137 \underline{62} 2018$ 2043_MOESM1 ESM.docx (921 kb)

Supplementary material 1 (DOCX $921 \mathrm{~kb})$

\section{References}

Aderolu AZ, Iyayp EA, Onilude AA (2007) Changes in nutritional value of rice husk during Trichoderma viride degradation. Bulgarian J Agric Sci 13(5):583-589

Google Scholar (http://scholar.google.com/scholar_lookup?

title=Changes\%2oin\%2onutritional\%20value\%20of\%2orice\%2ohusk\%2oduring\%20T richoderma\%2oviride\%2odegradation\&author=AZ.\%20Aderolu\&author=EA.\%20Iyay p\&author=AA.\%20Onilude\&journal=Bulgarian\%20J\%20Agric\%20Sci\&volume=13\&is sue $=5 \&$ pages $=583-589 \&$ publication_year $=2007$ )

Ahmedna M, Marshall WE, Husseiny AA, Rao RM, Goktepe I (2004) The use of nutshell carbons in drinking water filters for removal of trace metals. Water Res 38(4):1062-1068

CrossRef (https://doi.org/10.1016/j.watres.2003.10.047)

Google Scholar (http://scholar.google.com/scholar_lookup?

title=The\%2ouse\%20of\%2onutshell\%2ocarbons\%2oin\%2odrinking\%2owater\%2ofilt ers\%20for\%2oremoval\%20of\%20trace\%2ometals\&author=M.\%20Ahmedna\&author= WE.\%20Marshall\&author=AA.\%20Husseiny\&author=RM.\%20Rao\&author=I.\%20Go ktepe\&journal $=$ Water\%20Res\&volume $=38 \&$ \&issue $=4$ \&pages $=1062-$

1068\&publication_year=2004)

Awg-Adeni DS, Abd-Aziz S, Bujang K, Hassan MA (2010) Bioconversion of sago residue into value added products. Afr J Biotechnol 9(14):2016-2021

Google Scholar (http://scholar.google.com/scholar_lookup?

title=Bioconversion\%20of\%20sago\%2oresidue\%2ointo\%20value\%2oadded\%2oprodu cts\&author=DS.\%20Awg-Adeni\&author=S.\%20Abd-

Aziz\&author=K.\%2oBujang\&author=MA.\%2OHassan\&journal=Afr\%20J\%2OBiotechn ol\&volume=9\&issue $=14 \&$ pages $=2016-2021 \&$ publication_year $=2010$ ) 
Chandra TC, Mirna MM, Sudaryanto Y, Ismadji S (2007) Adsorption of basic dye onto activated carbon prepared from durian shell: studies of adsorption equilibrium and kinetics. Chem Eng J 127(1):121-129

CrossRef (https://doi.org/10.1016/j.cej.2006.09.011)

Google Scholar (http://scholar.google.com/scholar_lookup?

title=Adsorption\%20of\%2obasic\%20dye\%20onto\%20activated\%20carbon\%2oprepar ed\%2ofrom\%2odurian\%20shell\%3A\%20studies\%20of\%20adsorption\%20equilibrium \%20and\%2okinetics\&author=TC.\%20Chandra\&author=MM.\%20Mirna\&author=Y.\% 2oSudaryanto\&author $=$ S.\%2OIsmadji\&journal $=$ Chem\%20Eng\%20J\&volume=127\&iss ue $=1 \&$ pages $=121-129 \&$ publication_year $=2007)$

Foo KY, Hameed BH (2012) Coconut husk derived activated carbon via microwave induced activation: effects of activation agents, preparation parameters and adsorption performance. Chem Eng J 184:57-65

CrossRef (https://doi.org/10.1016/j.cej.2011.12.084)

Google Scholar (http://scholar.google.com/scholar_lookup?

title=Coconut\%2ohusk\%2oderived\%20activated\%2ocarbon\%20via\%2omicrowave\%2 oinduced\%20activation\%3A\%20effects\%20of\%20activation\%20agents\%2C\%2oprepa ration\%2oparameters\%20and\%20adsorption\%2operformance\&author=KY.\%20Foo\& author=BH.\%20Hameed\&journal=Chem\%20Eng\%20J\&volume $=184 \&$ pages $=57-$

65\&publication_year=2012)

Gao J, Kong D, Wang Y, Wu J, Sun S, Xu P (2013) Production of mesoporous activated carbon from tea fruit peel residues and its evaluation of methylene blue removal from aqueous solutions. Bioresource 8(2):2145-2160

CrossRef (https://doi.org/10.15376/biores.8.2.2145-2160)

Google Scholar (http://scholar.google.com/scholar_lookup?

title=Production\%20of\%2omesoporous\%20activated\%20carbon\%2ofrom\%20tea\%20 fruit\%2opeel\%20residues\%20and\%20its\%20evaluation\%20of\%2omethylene\%2oblue \%20removal\%2ofrom\%20aqueous\%20solutions\&author=J.\%20Gao\&author=D.\%20K ong\&author $=$ Y.\%20Wang\&author $=$ J.\%20Wu\&author $=$ S.\%20Sun\&author=P.\%20Xu\& journal $=$ Bioresource $\&$ volume $=8 \&$ issue $=2 \&$ pages $=2145$ -

2160\&publication_year=2013)

Hegde G, Abdul Manaf SA, Kumar A, Ali GA, Chong KF, Ngaini Z, Sharma KV (2015)

Biowaste sago bark based catalyst free carbon nanospheres: waste to wealth approach.

ACS Sustain Chem Eng 3(9):2247-2253

CrossRef (https://doi.org/10.1021/acssuschemeng.5boo517)

Google Scholar (http://scholar.google.com/scholar_lookup?

title=Biowaste\%20sago\%2obark\%2obased\%20catalyst\%2ofree\%20carbon\%2onanosp heres\%3A\%20waste\%20to\%20wealth\%20approach\&author=G.\%20Hegde\&author=S A.\%20Abdul\%2OManaf\&author=A.\%20Kumar\&author=GA.\%20Ali\&author=KF.\%20 Chong\&author=Z.\%20Ngaini\&author=KV.\%20Sharma\&journal=ACS\%2OSustain\%20 Chem\%20Eng\&volume=3\&issue $=9 \&$ pages $=2247-2253 \&$ publication_year $=2015$ )

Hesas RH, Arami-Niya A, Daud WMAW, Sahu JN (2013) Preparation and characterization of activated carbon from apple waste by microwave-assisted phosphoric acid activation: application in methylene blue adsorption. BioResour 8(2):2950-2966

Google Scholar (http://scholar.google.com/scholar_lookup?

title=Preparation\%20and\%20characterization\%20of\%20activated\%20carbon\%2ofro m\%20apple\%20waste\%20by\%2omicrowave-

assisted\%20phosphoric\%20acid\%20activation\%3A\%20application\%2oin\%2omethyle ne\%2oblue\%20adsorption\&author=RH.\%2OHesas\&author=A.\%2OArami-

Niya\&author=WMAW.\%20Daud\&author $=J N$.\%20Sahu\&journal=BioResour\&volume $=8 \&$ issue $=2 \&$ pages $=2950-2966 \&$ publication_year=2013） 
Ho YS, McKay G (1999) Pseudo-second order model for sorption processes. Process Biochem 34(5):451-465

CrossRef (https://doi.org/10.1016/Soo32-9592(98)oo112-5)

Google Scholar (http://scholar.google.com/scholar_lookup?title=Pseudosecond\%20order\%20model\%20for\%20sorption\%2oprocesses\&author=YS.\%20Ho\&a uthor $=$ G. $\% 20$ McKay\&journal $=$ Process $\% 20$ Biochem $\&$ volume $=34 \&$ issue $=5 \&$ pages $=451$ -465\&publication_year=1999)

Hu C, Li J, Zhou Y, Li M, Xue F, Li H (2009) Enhanced removal of methylene blue from aqueous solution by pummelo peel pretreated with sodium hydroxide. $J$ Health Sci 55(4):619-624

CrossRef (https://doi.org/10.1248/jhs.55.619)

Google Scholar (http://scholar.google.com/scholar_lookup?

title=Enhanced\%20removal\%20of\%20methylene\%2oblue\%2ofrom\%20aqueous\%20s olution\%2oby\%2opummelo\%2opeel\%2opretreated\%20with\%2osodium\%2ohydroxid e\&author=C.\%20Hu\&author=J.\%2OLi\&author=Y.\%20Zhou\&author=M.\%2OLi\&autho $\mathrm{r}=\mathrm{F} . \% 20 X u e \& a u t h o r=H . \% 20$ Li\&journal $=\mathrm{J} \% 2 \mathrm{OHealth} \% 20$ Sci\&volume $=55 \&$ issue $=4 \&$ pages $=619-624 \&$ publication $\_$year $=2009$ )

Kanawade SM, Gaikwad RW (2011) Removal of methylene blue from effluent by using activated carbon and water hyacinth as adsorbent. Int J Chem Eng Appl 2(5):317-319 Google Scholar (http://scholar.google.com/scholar_lookup?

title=Removal\%20of\%2omethylene\%2oblue\%2ofrom\%2oeffluent\%2oby\%2ousing\%2 oactivated\%20carbon\%20and\%20water\%2ohyacinth\%20as\%20adsorbent\&author=S M.\%2oKanawade\&author=RW.\%20Gaikwad\&journal=Int\%20J\%20Chem\%20Eng\%2 oAppl\&volume $=2 \&$ issue $=5 \&$ pages $=317-319 \&$ publication_year $=2011)$

Karnib M, Kabbani A, Holail H, Olama Z (2014) Heavy metals removal using activated carbon, silica and silica activated carbon composite. Energy Procedia 50:113-120

CrossRef (https://doi.org/10.1016/j.egypro.2014.06.014)

Google Scholar (http://scholar.google.com/scholar_lookup?

title=Heavy\%20metals\%2oremoval\%20using\%20activated\%20carbon\%2C\%2osilica\% 20and\%20silica\%20activated\%20carbon\%20composite\&author=M.\%20Karnib\&auth or=A.\%2OKabbani\&author=H.\%2OHolail\&author=Z.\%20Olama\&journal=Energy\%2O Procedia\&volume $=50 \&$ pages $=113-120 \&$ publication_year=2014）

Khaled A, El Nemr A, El-Sikaily A, Abdelwahab O (2009) Removal of Direct N Blue106 from artificial textile dye effluent using activated carbon from orange peel: adsorption isotherm and kinetic studies. J Hazard Mater 165(1):100-110

CrossRef (https://doi.org/10.1016/j.jhazmat.2008.09.122)

Google Scholar (http://scholar.google.com/scholar_lookup?

title=Removal\%20of\%2oDirect\%20N\%2oBlue-

106\%2ofrom\%20artificial\%2otextile\%2odye\%2oeffluent\%2ousing\%20activated\%20c arbon\%2ofrom\%20orange\%2opeel\%3A\%20adsorption\%2oisotherm\%20and\%2okinet ic\%20studies\&author=A.\%20Khaled\&author=A.\%20Nemr\&author=A.\%20El-

Sikaily\&author $=$ O.\%20Abdelwahab\&journal $=\mathrm{J} \% 20$ Hazard\%20Mater\&volume $=165$ \& is sue $=1 \&$ pages $=100-110 \&$ publication_year $=2009$ )

Khatod I (2013) Removal of methylene blue dye from aqueous solutions by neem leaf and orange peel powder. Int J ChemTech Res 5(2):572-577

Google Scholar (http://scholar.google.com/scholar_lookup?

title=Removal\%20of\%2omethylene\%2oblue\%20dye\%2ofrom\%20aqueous\%2osolutio ns\%2oby\%2oneem\%2oleaf\%20and\%20orange\%2opeel\%2opowder\&author=I.\%20Kh atod\&journal=Int\%20J\%20ChemTech\%20Res\&volume $=5 \&$ issue $=2 \&$ pages $=572-$

577\&publication_year=2013) 
Ludueña L, Fasce D, Alvarez VA, Stefani PM (2011) Nanocellulose from rice husk following alkaline treatment to remove silica. BioResour 6(2):1440-1453

Google Scholar (http://scholar.google.com/scholar_lookup?

title=Nanocellulose\%2ofrom\%2orice\%2ohusk\%2ofollowing\%2oalkaline\%2otreatmen t\%20to\%20remove\%20silica\&author=L.\%20Ludue\%C3\%B1a\&author=D.\%20Fasce\&a uthor=VA.\%20Alvarez\&author=PM.\%20Stefani\&journal=BioResour\&volume=6\&issu $\mathrm{e}=2$ \&pages $=1440-1453$ \&publication_year $=2011$ )

Martinez ML, Moiraghi L, Agnese M, Guzman C (2003) Making and some properties of activated carbon produced from agricultural industrial residues from Argentina.

Anales-AsociacionQuimica Argentina 91:103-108

Google Scholar (http://scholar.google.com/scholar_lookup?

title=Making\%20and\%20some\%2oproperties\%20of\%20activated\%2ocarbon\%2oprod uced\%2ofrom\%2oagricultural\%2oindustrial\%2oresidues\%2ofrom\%2oArgentina\&aut hor=ML.\%20Martinez\&author=L.\%20Moiraghi\&author=M.\%2oAgnese\&author=C.\% 20Guzman\&journal=Anales-

AsociacionQuimica\%20Argentina\&volume $=91 \&$ pages $=103-$

108\&publication_year=2003)

Moreno-Castilla C, Carrasco-Marı́ n F, Lopez-Ramon MV, Alvarez-Merino MA (2001) Chemical and physical activation of olive-mill waste water to produce activated

carbons. Carbon 39(9):1415-1420

CrossRef (https://doi.org/10.1016/Sooo8-6223(00)00268-2)

Google Scholar (http://scholar.google.com/scholar_lookup?

title=Chemical\%20and\%2ophysical\%2oactivation\%20of\%20olive-

mill\%20waste\%20water\%20to\%2oproduce\%20activated\%20carbons\&author=C.\%20

Moreno-Castilla\&author=F.\%2oCarrasco-

Mar\%C4\%B1\%CC\%81n\&author=MV.\%2oLopez-Ramon\&author=MA.\%20Alvarez-

Merino\&journal $=$ Carbon\&volume $=39$ \&issue $=9$ \&pages $=1415^{-}$

1420\&publication_year=2001)

Ngaini Z, Rahman KAAA, Shaari N, Hussain H, Sundin N, Jingxin T, Lawai V (2013) Production of fire-retardant sound-absorbing panels from sago waste. J Trop For Sci 25(4):510-515

Google Scholar (http://scholar.google.com/scholar_lookup?

title=Production\%20of\%2ofire-retardant\%20sound-

absorbing\%2opanels\%2ofrom\%20sago\%20waste\&author=Z.\%20Ngaini\&author=KA

AA.\%2ORahman\&author=N.\%2OShaari\&author $=$ H.\%2OHussain\&author=N.\%2OSund in\&author=T.\%20Jingxin\&author=V.\%20Lawai\&journal=J\%20Trop\%20For\%20Sci\& volume $=25 \&$ issue $=4 \&$ pages $=510-515 \&$ publication_year $=2013$ )

Ngaini Z, Noh F, Wahi R (2014) Esterified sago waste for engine oil removal in aqueous environment. Environ Technol 35(22):2761-2766

CrossRef (https://doi.org/10.1080/09593330.2014.920051)

Google Scholar (http://scholar.google.com/scholar_lookup?

title=Esterified \%20sago\%20waste\%20for\%2oengine\%20oil\%2oremoval\%2oin\%20aq ueous\%20environment\&author=Z.\%20Ngaini\&author=F.\%20Noh\&author=R.\%20Wa hi\&journal=Environ\%20Technol\&volume $=35$ \&issue $=22 \&$ pages $=2761-$

2766\&publication_year $=2014$ )

Ngaini Z, Noh F, Wahi R (2017) Facile sorbent from esterified cellulosic sago waste for engine oil removal in marine environment. Int J Environ Sci Technol.

https://doi.org/10.1007/s13762-017-1389-9 (https://doi.org/10.1007/s13762-017-

1389-9)

CrossRef (https://doi.org/10.1007/s13762-017-1389-9)

Google Scholar (http://scholar.google.com/scholar_lookup?

title=Facile\%20sorbent\%2ofrom\%2oesterified\%2ocellulosic\%2osago\%20waste\%2ofo 
r\%20engine\%20oil\%2oremoval\%20in\%2omarine\%2oenvironment\&author=Z.\%20Ng aini\&author=F.\%20Noh\&author=R.\%20Wahi\&journal=Int\%20J\%20Environ\%20Sci \%20Technol\&publication_year=2017\&doi=10.1007\%2Fs13762-017-1389-9)

Nishihara H, Fukura Y, Inde K, Tsuji K, Takeuchi M, Kyotani T (2008) Carbon-coated mesoporous silica with hydrophobicity and electrical conductivity. Carbon 46(1):48-

53

CrossRef (https://doi.org/10.1016/j.carbon.2007.10.024)

Google Scholar (http://scholar.google.com/scholar_lookup?title=Carbon-

coated\%20mesoporous\%2osilica\%20with\%2ohydrophobicity\%20and\%2oelectrical\%2 oconductivity\&author=H.\%20Nishihara\&author=Y.\%20Fukura\&author=K.\%20Inde\& author=K.\%20Tsuji\&author=M.\%20Takeuchi\&author=T.\%20Kyotani\&journal=Carbo n\&volume $=46 \&$ issue $=1 \&$ pages $=48-53 \&$ publication_year $=2008$ )

Novak JM, Lima I, Xing B, Gaskin JW, Steiner C, Das KC, Ahmedna M, Rehrah D, Watts DW, Busscher WJ, Schomberg H (2009) Characterization of designer biochar produced at different temperatures and their effects on a loamy sand. Ann Environ Sci 3(1):195-206

Google Scholar (http://scholar.google.com/scholar_lookup?

title=Characterization\%20of\%2odesigner\%2obiochar\%2oproduced\%20at\%2odifferen t\%2otemperatures\%20and\%20their\%2oeffects\%20on\%20a\%2oloamy\%20sand\&auth or=JM.\%20Novak\&author=I.\%20Lima\&author=B.\%20Xing\&author=JW.\%20Gaskin \&author=C.\%20Steiner\&author=KC.\%20Das\&author=M.\%20Ahmedna\&author=D.\% 20Rehrah\&author=DW.\%20Watts\&author=WJ.\%20Busscher\&author=H.\%20Schom berg\&journal=Ann\%20Environ\%20Sci\&volume=3\&issue $=1$ \&pages $=195-$

206\&publication_year=2009)

Patil S, Renukdas S, Patel N (2011) Removal of methylene blue, a basic dye from aqueous solutions by adsorption using teak tree (Tectona grandis) bark powder. Int $\mathrm{J}$ Environ Sci 1(5):711-725

Google Scholar (http://scholar.google.com/scholar_lookup?

title=Removal\%20of\%2omethylene\%2oblue\%2C\%20a\%2obasic\%2odye\%2ofrom\%20 aqueous\%20solutions\%20by\%20adsorption\%20using\%20teak\%20tree\%20\%28Tecto na\%20grandis\%29\%2obark\%2Opowder\&author=S.\%2OPatil\&author=S.\%20Renukda s\&author=N.\%20Patel\&journal=Int\%20J\%20Environ $\% 20$ Sci\&volume $=1 \& i s s u e=5 \& p a$ ges $=711-725 \&$ publication_year $=2011$ )

Rahman MA, Amin SR, Alam AS (2012) Removal of methylene blue from waste water using activated carbon prepared from rice husk. Dhaka Univ J Sci 6o(2):185-189 Google Scholar (http://scholar.google.com/scholar_lookup?

title=Removal\%20of\%20methylene\%20blue\%2ofrom\%20waste\%20water\%20using\% 20activated\%20carbon\%2oprepared\%20from\%2orice\%2ohusk\&author=MA.\%2oRah man\&author=SR.\%20Amin\&author=AS.\%20Alam\&journal=Dhaka\%20Univ\%20J\%2 oSci\&volume $=60 \&$ issue $=2$ \&pages $=185-189$ \&publication_year $=2012$ )

Shendkar CD, Torane RC, Mundhe KS, Lavate SM, Pawar AB, Deshpande NR (2013) Characterization and application of activated carbon prepared from waste weed. Int $J$ Pharm Pharm Sci 5:527-529

Google Scholar (http://scholar.google.com/scholar_lookup?

title=Characterization\%20and\%20application\%20of\%20activated\%2ocarbon\%2oprep ared\%2ofrom\%20waste\%20weed\&author=CD.\%20Shendkar\&author=RC.\%20Torane \&author=KS.\%20Mundhe\&author=SM.\%2OLavate\&author=AB.\%20Pawar\&author $=\mathrm{N}$ R.\%20Deshpande\&journal=Int\%20J\%20Pharm\%20Pharm\%20Sci\&volume=5\&pages $=527-529 \&$ \&ublication_year $=2013$ )

Sisman CB, Gezer E, Kocaman I (2011) Effects of organic waste (rice husk) on the concrete properties for farm buildings. Bulgarian J Agric Sci 17(1):40-48 
Google Scholar (http://scholar.google.com/scholar_lookup?

title=Effects\%20of\%20organic\%20waste\%20\%28rice\%2ohusk\%29\%20on\%20the\%20 concrete\%20properties\%2ofor\%2ofarm\%2obuildings\&author=CB.\%20Sisman\&autho $\mathrm{r}=\mathrm{E} . \% 20$ Gezer\&author=I.\%20Kocaman\&journal=Bulgarian\%20J\%20Agric\%20Sci\&v olume $=17$ \&issue $=1 \&$ pages $=40-48 \&$ publication_year $=2011$ )

Skubiszewska-Zieba J, Charmas B, Leboda R, Staszczuk P, Kowalczyk P, Oleszczuk P (2003) Effect of hydrothermal modification on the porous structure and thermal properties of carbon-silica adsorbents (carbosils). Mater Chem Phys 78(2):486-494

CrossRef (https://doi.org/10.1016/So254-0584(02)00357-7)

Google Scholar (http://scholar.google.com/scholar_lookup?

title=Effect\%20of\%20hydrothermal\%20modification\%20on\%20the\%2oporous\%20st ructure\%20and\%20thermal\%2oproperties\%20of\%20carbon\%E2\%80\%93silica\%2oad sorbents\%20\%28carbosils\%29\&author=J.\%20Skubiszewska-

Zieba\&author=B.\%20Charmas\&author=R.\%20Leboda\&author=P.\%20Staszczuk\&aut hor=P.\%20Kowalczyk\&author=P.\%2oOleszczuk\&journal=Mater\%2oChem\%2oPhys\& volume $=78$ \&issue $=2 \&$ pages $=486-494 \&$ publication_year $=2003$ )

Tan IAW, Ahmad AL, Hameed BH (2008) Optimization of preparation conditions for activated carbons from coconut husk using response surface methodology. Chem Eng J 137(3):462-470

CrossRef (https://doi.org/10.1016/j.cej.2007.04.031)

Google Scholar (http://scholar.google.com/scholar_lookup?

title=Optimization\%20of\%2opreparation\%20conditions\%2ofor\%2oactivated\%20carb ons\%2ofrom\%20coconut\%2ohusk\%20using\%2oresponse\%20surface\%2omethodolog y\&author=IAW.\%20Tan\&author=AL.\%2OAhmad\&author=BH.\%20Hameed\&journal= Chem\%2oEng\%20J\&volume $=137$ \&issue $=3 \&$ pages $=462-$

470\&publication_year=2008)

Tan XW, Romainor ANB, Chin SF, Ng SM (2014) Carbon dots production via pyrolysis of sago waste as potential probe for metal ions sensing. J Anal Appl Pyrolysis 105:157165

CrossRef (https://doi.org/10.1016/j.jaap.2013.11.001)

Google Scholar (http://scholar.google.com/scholar_lookup?

title=Carbon\%20dots\%2oproduction\%20via\%2opyrolysis\%20of\%20sago\%20waste\% 20as\%2opotential\%2oprobe\%2ofor\%2ometal\%2oions\%2osensing\&author=XW.\%20 Tan\&author=ANB.\%20Romainor\&author=SF.\%20Chin\&author=SM.\%20Ng\&journal $=$ J\%20Anal\%20Appl\%20Pyrolysis\&volume $=105$ \&pages $=157-$

165\&publication_year=2014)

Thirumalisamy S, Subbian M (2010) Removal of methylene blue from aqueous solution by activated carbon prepared from the peel of Cucumis sativa fruit by adsorption. BioResour 5(1):419-437

Google Scholar (http://scholar.google.com/scholar_lookup?

title=Removal\%20of\%2omethylene\%2oblue\%2ofrom\%2oaqueous\%2osolution\%2oby \%20activated\%2ocarbon\%2oprepared\%2ofrom\%20the\%2opeel\%20of\%20Cucumis\% 20sativa\%2ofruit\%2oby\%2oadsorption\&author=S.\%2oThirumalisamy\&author=M.\%2 oSubbian\&journal $=$ BioResour\&volume $=5$ \&issue $=1$ \&pages $=419-$

437\&publication_year=2010)

Valle-Vigón P, Sevilla M, Fuertes AB (2012) Sulfonated mesoporous silica-carbon composites and their use as solid acid catalysts. Appl Surf Sci 261:574-583

CrossRef (https://doi.org/10.1016/j.apsusc.2012.08.059)

Google Scholar (http://scholar.google.com/scholar_lookup?

title=Sulfonated\%20mesoporous\%20silica\%E2\%80\%93carbon\%20composites\%20an d\%20their\%20use\%20as\%20solid\%20acid\%20catalysts\&author=P.\%20Valle- 
Vig\%C3\%B3n\&author=M.\%20Sevilla\&author=AB.\%2oFuertes\&journal=Appl\%2oSurf \%20Sci\&volume $=261 \&$ pages $=574-583 \&$ publication_year $=2012$ )

Vieira MGA, de Almeida Neto AF, Da Silva MGC, Carneiro CN, Melo Filho AA (2014) Adsorption of lead and copper ions from aqueous effluents on rice husk ash in a dynamic system. Braz J Chem Eng 31(2):519-529

CrossRef (https://doi.org/10.1590/0104-6632.20140312s00002103)

Google Scholar (http://scholar.google.com/scholar_lookup?

title=Adsorption\%20of\%2olead\%20and\%20copper\%20ions\%2ofrom\%20aqueous\%2 oeffluents\%20on\%2orice\%2ohusk\%20ash\%20in\%20a\%20dynamic\%20system\&auth or=MGA.\%20Vieira\&author=AF.\%20Almeida\%20Neto\&author=MGC.\%20Silva\&auth or=CN.\%20Carneiro\&author=AA.\%20Melo\%20Filho\&journal=Braz\%20J\%20Chem\% 20Eng\&volume=31\&issue $=2 \&$ pages $=519-529 \&$ publication_year $=2014 ）$

Wahi R, Senghie H (2010) The use of microwave derived activated carbon for removal of heavy metal in aqueous solution. J Sci Technol 3(1):97-108

Google Scholar (http://scholar.google.com/scholar_lookup?

title=The\%20use\%20of\%2omicrowave\%2oderived\%20activated\%2ocarbon\%2ofor\%2 oremoval\%20of\%2oheavy\%2ometal\%20in\%20aqueous\%20solution\&author=R.\%20

Wahi\&author $=$ H.\%2oSenghie\&journal $=J \% 20$ Sci\%20Technol\&volume $=3 \&$ issue $=1 \&$ pa ges=97-108\&publication_year $=2010$ )

Wahi R, Ngaini Z, Jok VU (2009) Removal of mercury, lead and copper from aqueous solution by activated carbon of palm oil empty fruit bunch. World Appl Sci J

5(84):84-91

Google Scholar (http://scholar.google.com/scholar_lookup?

title=Removal\%20of\%2omercury\%2C\%2olead\%20and\%2ocopper\%2ofrom\%20aqueo us\%20solution\%2oby\%20activated\%20carbon\%20of\%2opalm\%20oil\%20empty\%2of ruit\%2obunch\&author=R.\%20Wahi\&author=Z.\%20Ngaini\&author=VU.\%2OJok\&jour nal=World\%20Appl\%20Sci\%20J\&volume $=5$ \&issue $=84$ \&pages $=84-$

91\&publication_year=2009)

Yan T, Wang L (2013) Adsorptive removal of methylene blue from aqueous solution by spent mushroom substrate: equilibrium, kinetics, and thermodynamics. BioResour

$8(3): 4722-4734$

CrossRef (https://doi.org/10.15376/biores.8.3.4722-4734)

Google Scholar (http://scholar.google.com/scholar_lookup?

title=Adsorptive\%2oremoval\%20of\%20methylene\%2oblue\%2ofrom\%2oaqueous\%20 solution\%2oby\%2ospent\%2omushroom\%20substrate\%3A\%2oequilibrium\%2C\%2oki netics\%2C\%20and\%2othermodynamics\&author=T.\%20Yan\&author=L.\%2oWang\&jo urnal $=$ BioResour\&volume $=8 \&$ issue $=3 \&$ pages $=4722-4734 \&$ publication_year $=2013$ )

Zohre S, Ataallah SG, Mehdi A (2010) Experimental study of methylene blue adsorption from aqueous solutions onto carbon nano tubes. Int $J$ Water Resour Environ Eng 2(2):16-28

Google Scholar (http://scholar.google.com/scholar_lookup?

title=Experimental\%20study\%20of\%2omethylene\%2oblue\%20adsorption\%2ofrom\% 20aqueous\%20solutions\%20onto\%20carbon\%20nano\%2otubes\&author=S.\%2OZohre \&author=SG.\%20Ataallah\&author=A.\%20Mehdi\&journal=Int\%20J\%20Water\%20Re sour\%20Environ\%20Eng\&volume $=2$ \&issue $=2$ \&pages $=16$ 28\&publication_year=2010)

\section{Copyright information}

(C) Islamic Azad University (IAU) 2018 


\section{About this article}

Cite this article as:

Rajan, Y., Ngaini, Z. \& Wahi, R. Int. J. Environ. Sci. Technol. (2018). https://doi.org/10.1007/s13762-0182043-X

- Received 20 September 2017

- Revised o3 September 2018

- Accepted 01 October 2018

- First Online og October 2018

- DOI https://doi.org/10.1007/s13762-018-2043-x

- Publisher Name Springer Berlin Heidelberg

- Print ISSN 1735-1472

- Online ISSN 1735-2630

- About this journal

- $\underline{\text { Reprints and Permissions }}$

- Published on behalf of the Science and Research Branch of the Islamic Azad University

Published in cooperation with

Published on behalf of the Science and Research Branch of the Islamic Azad University

\section{Personalised recommendations}

\section{SPRINGER NATURE}

(C) 2018 Springer Nature Switzerland AG. Part of $\underline{\text { Springer Nature. }}$

Not logged in Universiti Malaysia Sarawak (3000088070) - 6824 SpringerLink Malaysia LNCS Consortium (3000122125) - 6816 SpringerLink Malaysia eJournal Consortium - Higher Education (3000155375) - 4973 SpringerLink Malaysia eBook Consortium-2010 copyright-year titl (3000164962) - 8326 SpringerLink Malaysia LNCS Consortium (3000254928) - 8354 Springerlink Malaysia consortium (3000519906) - 10122 SpringerLink Malaysia eJourna Consortium - Higher Education (3000716851) - SpringerLink Malaysia eJournal Consortium Higher Education (3000916360) - 12471 SpringerLink Malaysia LNCS Consortium (3000969622) - 15828 SpringerLink Malaysia LNCS Consortium (3991448967) - SpringerLink Malaysia LNCS Consortium 2015 (3991461284) 49.50.236.221 